\title{
Brève histoire de la collection Gazola de poissons fossiles éocènes du Monte Bolca (Italie) conservée au Muséum national d'Histoire naturelle, Paris
}

\author{
Jean GAUDANT \\ Muséum national d'Histoire naturelle, \\ Département Histoire de la Terre, USM203, UMR 7207 CNRS, \\ case postale 38, 57 rue Cuvier, F-75231 Paris cedex 05 (France) \\ jean.gaudant@orange.fr
}

MOTS CLÉS

collection paléontologique, poissons fossiles,

Éocène,

Italie,

XVIIIe siècle,

XIXe siècle.

KEY WORDS

palaeontological

collection,

fossil fishes,

Eocene,

Italy,

18 th century,

19 th century.
Gaudant J. 2011. - Brève histoire de la collection Gazola de poissons fossiles éocènes du Monte Bolca (Italie) conservée au Muséum national d'Histoire naturelle, Paris. Geodiversitas 33 (4): 637-647. DOI: 10.5252/g2011n4a5.

\section{RÉSUMÉ}

L'histoire de la collection Gazola de poissons fossiles du Monte Bolca (Italie) est généralement connue de manière incomplète en faisant seulement référence à la réquisition imposée, en mai 1797, par des commissaires qui accompagnaient les troupes françaises qui occupaient Vérone. Toutefois, l'étude des sources manuscrites a permis de montrer que Giovanni Battista Gazola a longuement séjourné à Paris en 1803 et qu'il offrit aux administrateurs du Muséum national d'Histoire naturelle de faire don de nouveaux spécimens destinés à compléter la partie de sa collection qui lui avait été confisquée cinq ans plus tôt.

\footnotetext{
ABSTRACT

Short history of the Gazola's collection of fossil fishes from the Eocene of Monte Bolca (Italy) housed in the Muséum national d'Histoire naturelle, Paris.

The history of Gazola's collection of fossil fishes from Monte Bolca (Italy) is generally incompletely known as reference is only made to the requisitioning of this collection, in May 1797, by commissioners who accompanied the French troops which occupied Verona. However, the study of manuscript sources has allowed to show that Giovanni Battista Gazola stayed in Paris for a long time during the year 1803 and that, on the 10th of May (20 Floreal an 11), he wrote a letter to the administrators of the french Muséum national d'Histoire naturelle, for proposing them to offer new specimens that could complete the part of his collection which had been seized five years earlier.
} 


\section{INTRODUCTION}

Homme des Lumières, le comte Giovanni Battista Gazola (1757-1834) avait commencé vers 1784 à constituer une collection de poissons fossiles du célèbre gisement fossilifère du Monte Bolca, dont il avait acquis partiellement la propriété en 1787 (Frigo \& Sorbini 1997). Quatre ans plus tard, il s'était porté acquéreur dans des conditions favorables rapportées par Nicolis (1893) du célèbre cabinet de Vincenzo Bozza, dont Giovanni Serafino Volta avait, dans l'intervalle, dressé le catalogue (Volta 1789). L'année suivante fut fondée une société qui s'était donné pour objectif de publier en italien et en latin la description de ces poissons dans un ouvrage intitulé Ittiolitologia Veronese ou Description du célèbre Cabinet de Bozza et d'autres Musées réputés d'ichthyolites de Vérone... comme en témoigne un prospectus imprimé en 1793 sous le titre Agli amatori della Storia naturale. Enfin, commença en 1796 la publication par livraisons de ce grand ouvrage qui débuta par l'impression de textes généraux d'introduction, d'une description topographique du site de la Lastrara, connue aujourd'hui sous le nom de Pesciara, de généralités sur la constitution des monts de Vestena et Bolca et enfin de considérations sur leur formation et sur l'origine des ichthyolithes. Ce n'est qu'après la réquisition du cabinet de Giovanni Battista Gazola que débuta la publication de la seconde partie de l'Ittiolitologia Veronese qui commence par la description du cabinet de Vincenzo Bozza, suivie par celle du musée de Gazola dont la confiscation partielle avait été rapidement compensée par l'acquisition de «la nombreuse collection de poissons fossiles laissée par le Co. Ignazio Ronconi».

\section{LA CONFISCATION DU CABINET GAZOLA}

Le 1er juin 1796, les troupes françaises firent leur entrée à Vérone d'où elles furent brièvement chassées par les Autrichiens du 30 juillet au 8 août. Dans les mois qui suivirent, les victoires d'Arcole (15-17 novembre 1796), puis de Rivoli (12-14 janvier 1797) et la capitulation de Mantoue (le 2 février 1797), assurèrent la domination des armées françaises sur la partie occidentale de la Vénétie. Cependant, les partisans de la République de Venise ne désarmaient pas. Ils se soulevèrent à Vérone le 17 avril 1797, aux cris de "Viva San Marco». Au cours de cet épisode sanglant qualifié de «Pâques véronaises", plusieurs dizaines de soldats français furent massacrés, les autres se réfugiant dans les points fortifiés de la ville (Cipolla 1954). Quatre jours furent nécessaires aux troupes françaises appelées en renfort pour maîtriser l'insurrection. La répression qui s'ensuivit fut féroce. Le général Augereau prit le commandement de la place de Vérone le 16 floréal an 5 (5 mai 1797). Dès le lendemain (17 floréal an 5), Bonaparte prit à Milan un arrêté qui lui ordonnait d'exécuter un ensemble de mesures destinées à sanctionner la ville de Vérone. Ces sanctions prévoyaient entre autres une lourde imposition financière au bénéfice de la République et des soldats français concernés, la confiscation de l'argenterie des églises et des bâtiments publics, la réquisition des chevaux et la mise à disposition de cuir et de tissus destinés à la fabrication d'uniformes pour les troupes françaises. Son article XI stipulait en outre que "Tous les tableaux, collections de plantes, de coquillages, etc. qui appartiendraient soit à la ville, soit aux particuliers seront confisqués au profit de la république» (Daru 1821: 329). Les troupes françaises étaient accompagnées de commissaires scientifiques et artistiques ayant pour mission d'inventorier les biens culturels susceptibles d'être réquisitionnés en vue de leur transfert en France. C'est ainsi que le chimiste Claude Berthollet (1748-1822) fut chargé de dresser la liste des objets susceptibles d'enrichir les musées français et la Bibliothèque nationale. Comme en fait foi un procès-verbal de réquisition conservé aux Archives nationales de France (Fig. 2) et dont la copie subsiste dans l'Archivio di Stato de Vérone, une délégation de la "Commission des Arts» conduite par Berthollet se présenta le 28 floréal an 5 (17 mai 1797) à la porte du palais Gazola dans lequel deux pièces avaient été aménagées pour l'exposition des plus beaux spécimens de poissons et végétaux pétrifiés du Monte Bolca. Ces fossiles furent « jugés dignes d'être portés dans le Muséum de Paris où manque cette collection ». Il s'agit donc bel et bien d'une confiscation. 


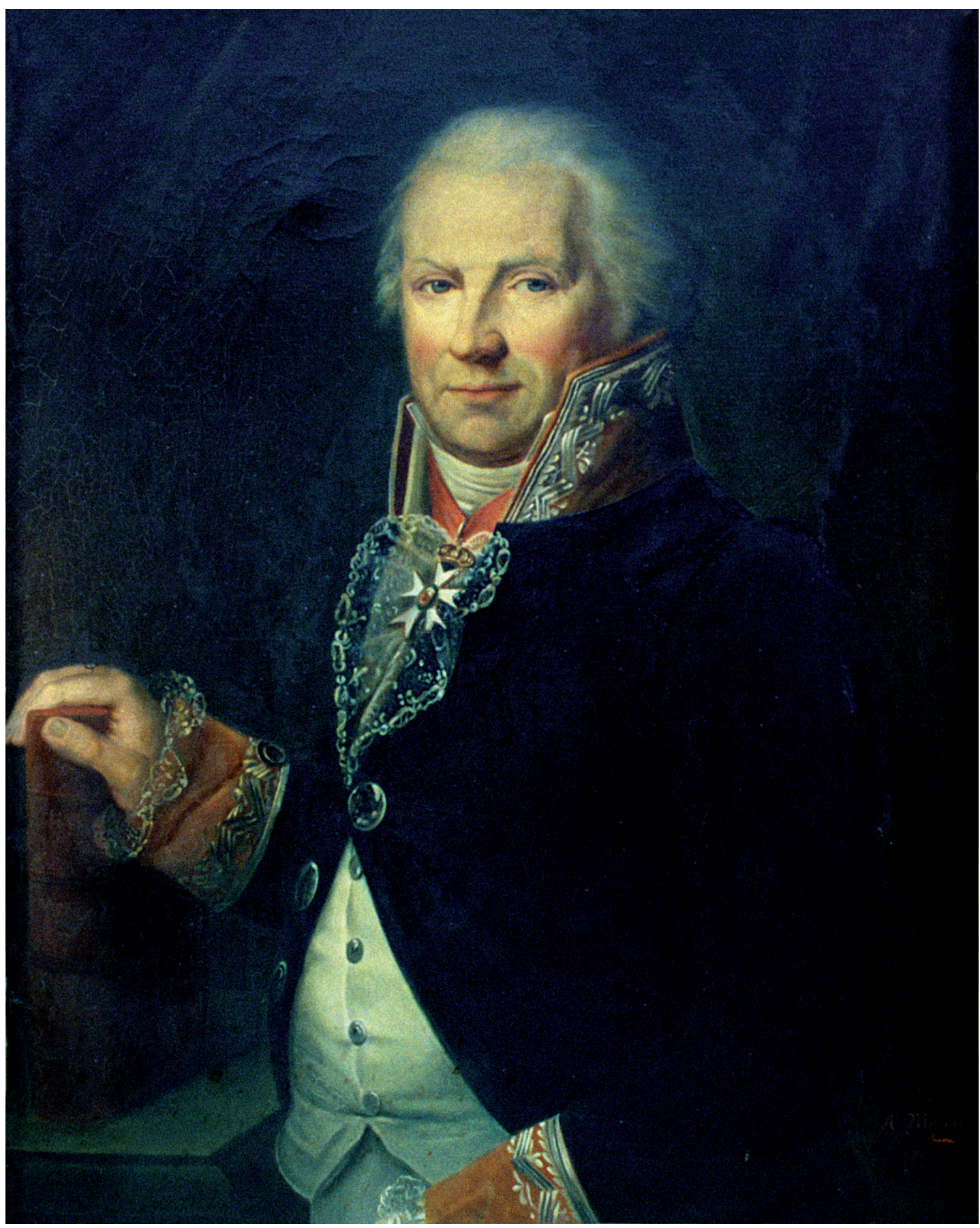

FIG. 1. - Copie moderne du portrait de Giovanni Battista Gazola (1757-1834) peint par H. N. Vangorp (cliché conservé dans l'Archivio del Museo di Storia naturale di Verona). Photographie: Archives du Musée d'Histoire naturelle de Vérone. Autorisation: B. Burato. 

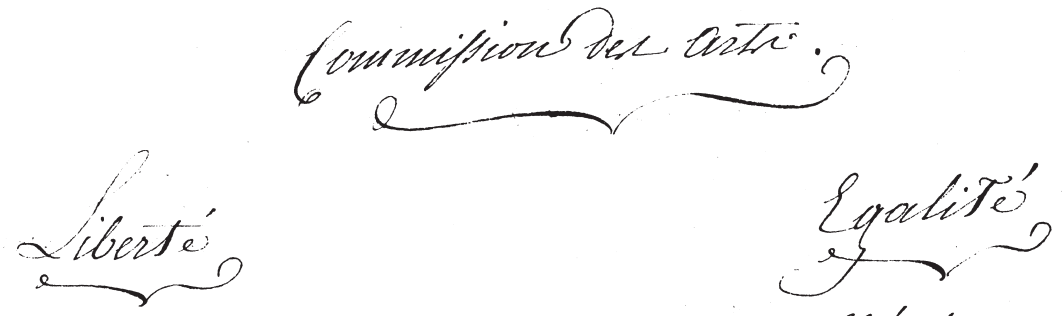

6 jounthui viugt hict flocent on is.

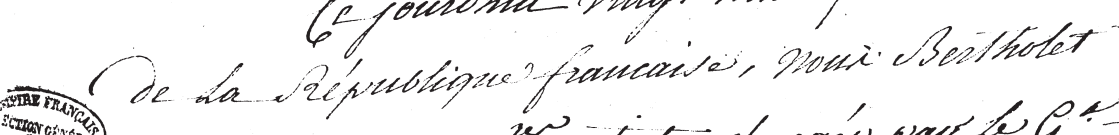

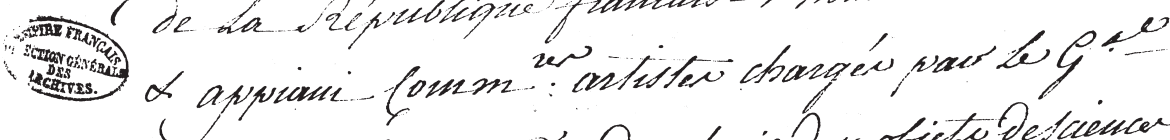

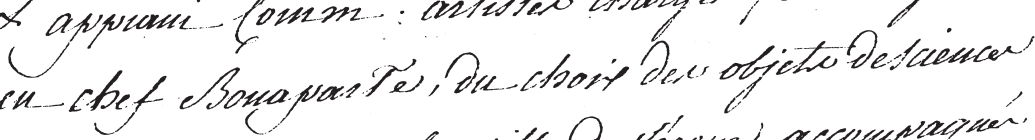

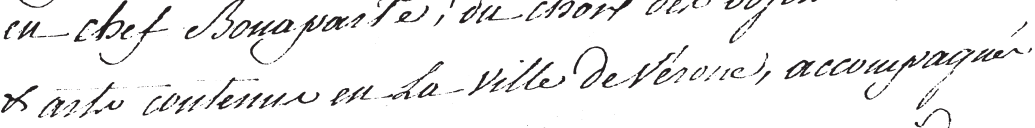

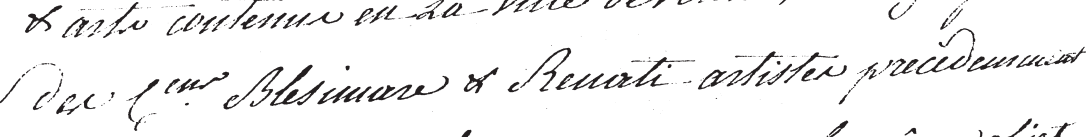

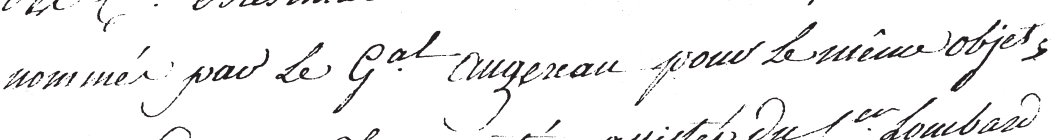

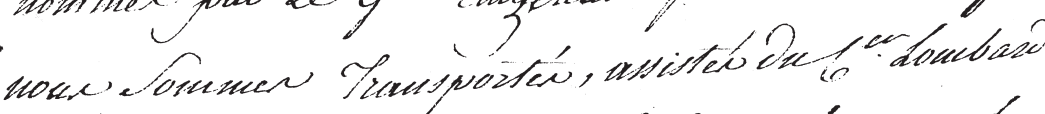

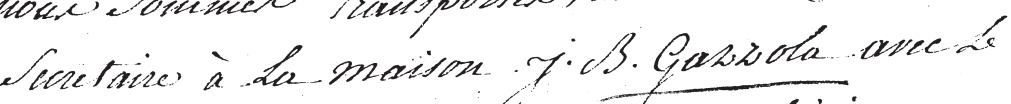

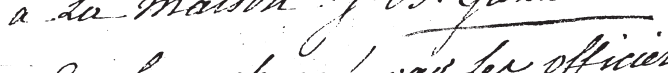
Ger pietro Gevota changé pais bev officien -

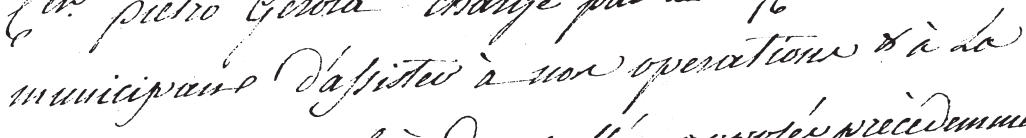

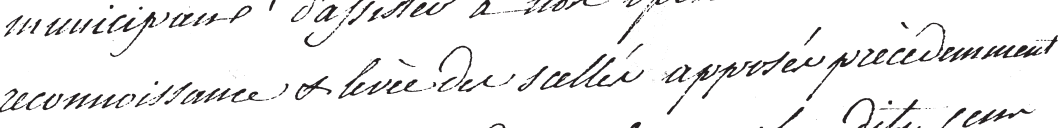

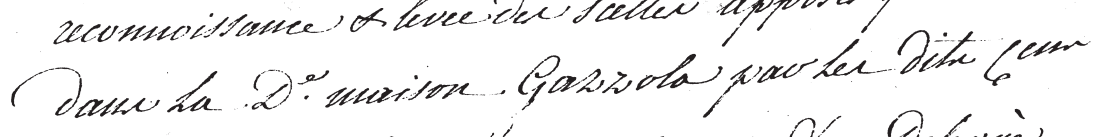

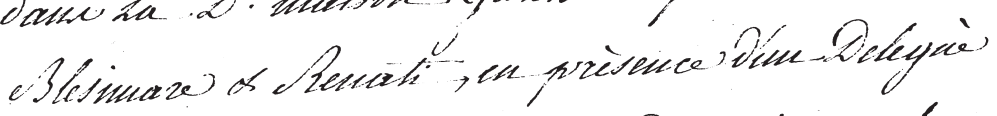

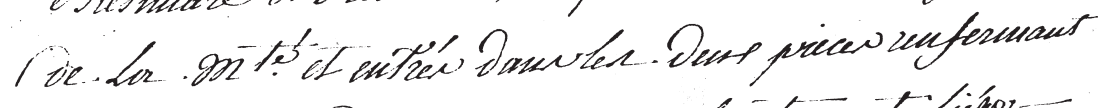

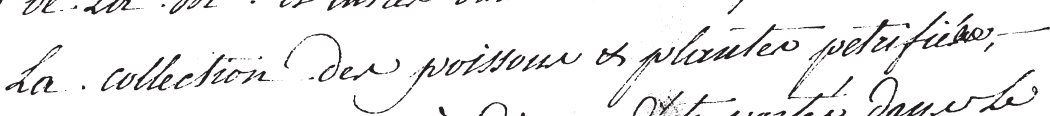

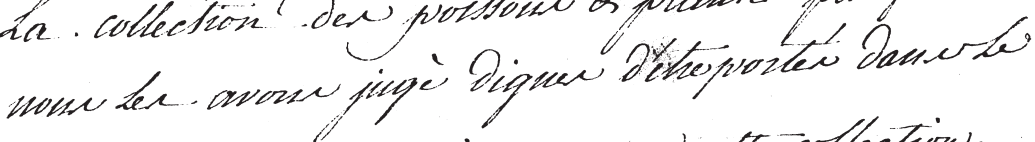

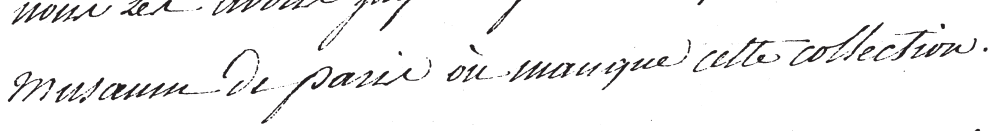

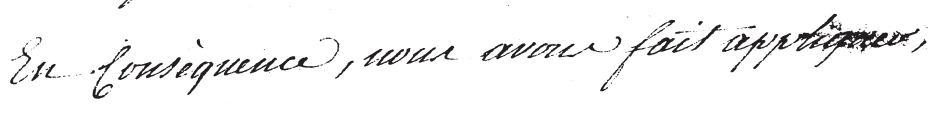


La collection Gazola de poissons fossiles éocènes du Monte Bolca (Italie)

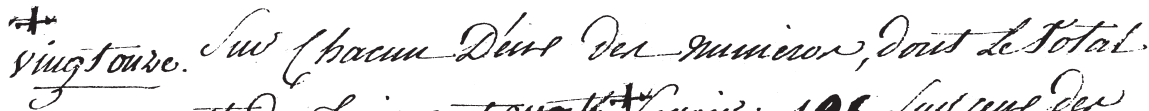

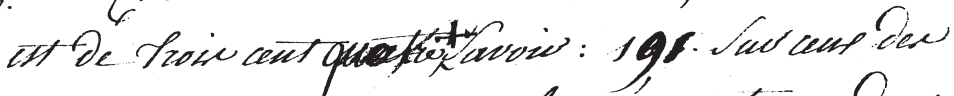

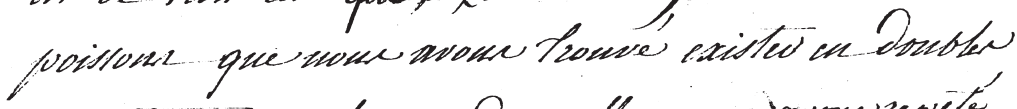

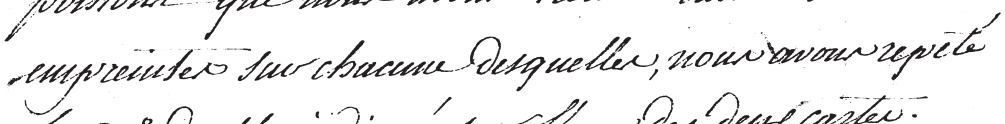

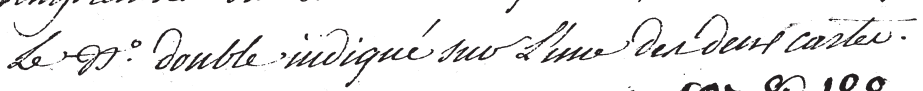

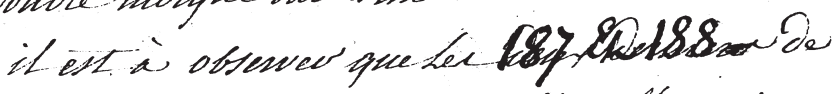

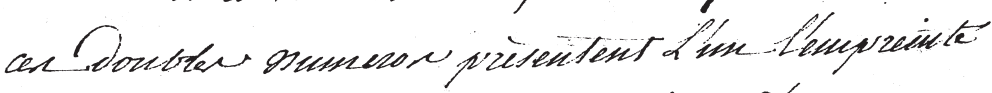

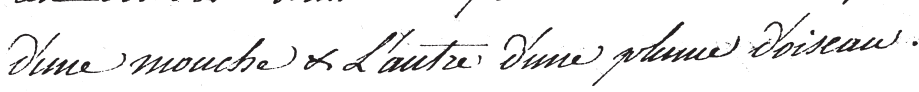

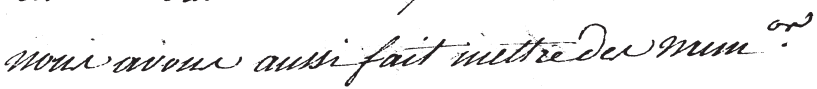

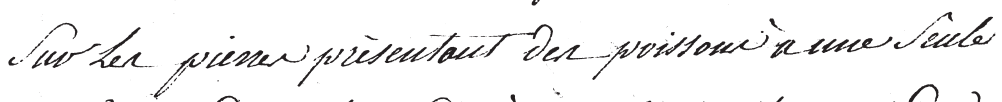

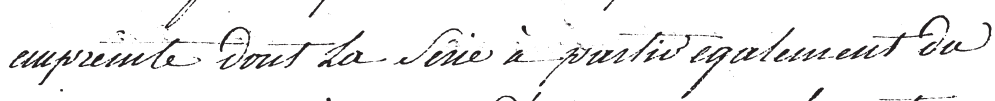

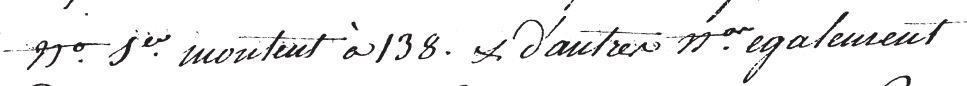

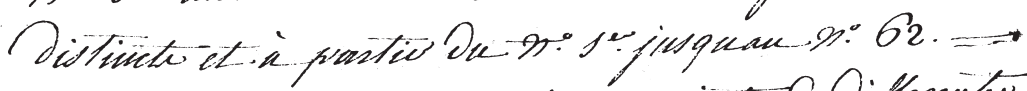

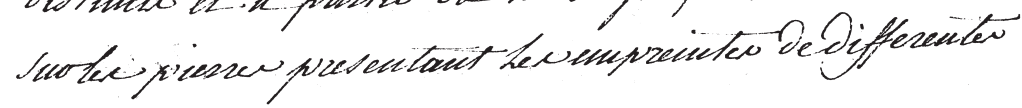
stantes of faillew.

Sesquete objete some avome fait

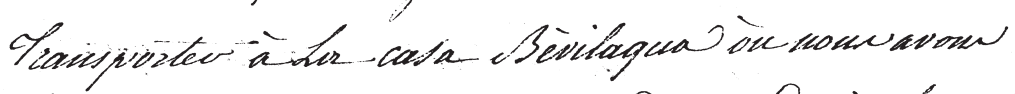

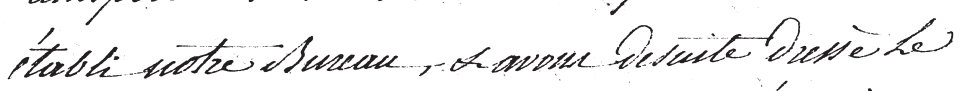

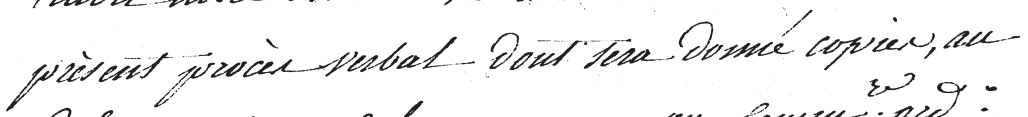

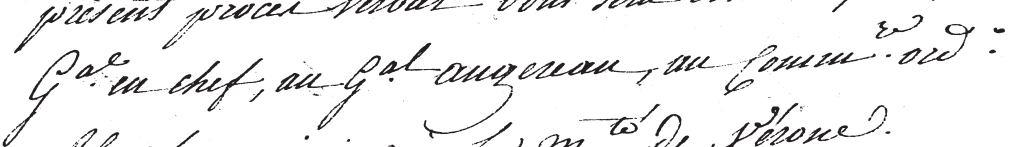

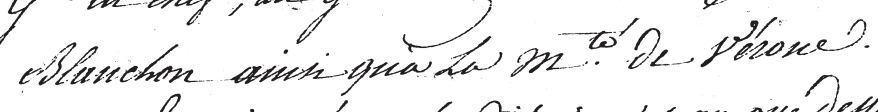

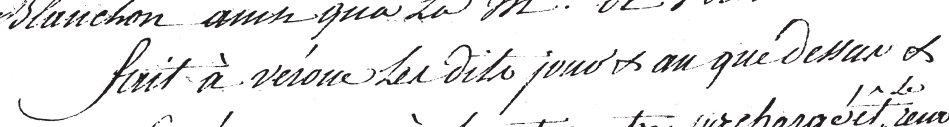

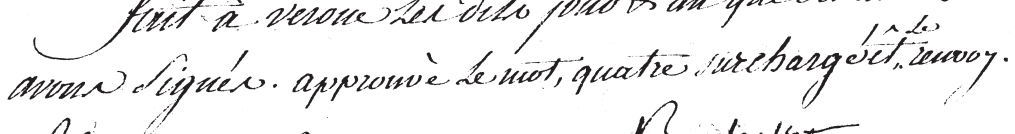
Bresimare, Renán

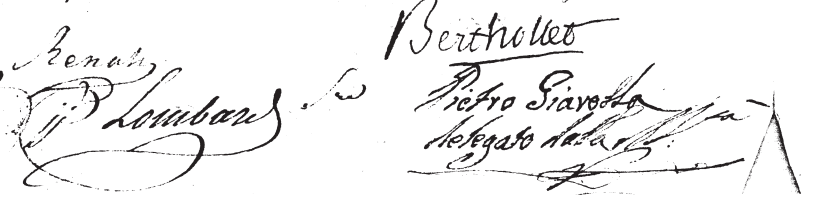

FIG. 2. - Suite.

GEODIVERSITAS • $2011 \bullet 33$ (4)

641 
Le nombre des fossiles réquisitionnés par les commissaires français est indiqué dans une lettre d'André Thouin, adressée de Livourne le 29 prairial an 5 (17 juin 1797) aux professeurs administrateurs du Muséum national d'Histoire naturelle (Archives nationales, Paris, dossier AJ/15/836; Fig. 2). On y lit qu' "elle est composée $1^{\circ}$ de 191 pièces doubles présentant les deux moitiés de chaque poisson, par conséquent 282 échantillons [heureusement, Thouin n’enseignait pas les mathématiques!]. $2^{\circ}$ de 138 pièces simples qui n'offrent l'empreinte que d'un côté. $3^{\circ}$ de 20 effigies de plantes doubles tirées de la même mine et formant 40 échantillons. $4^{\circ}$ et enfin 38 figures simples, de différentes plantes étrangères» (Fig. 3).

Les procès-verbaux des assemblées des professeurs du Muséum permettent de suivre le transport de ces fossiles. Ainsi, le 24 messidor de l'an 5 (12 juillet 1797) annonce-t-on que «Le citoyen Thouin professeur écrit de Livourne qu'on vient d'achepter [sic] et d'encaisser pour le Museum d'histoire naturelle une collection de poissons pétrifiés fort considérable, tiré [sic] de la montagne de Veronne [sic]» (Il s'agit de la lettre mentionnée ci-dessus). Effectivement, dans une lettre adressée de Livourne le 29 prairial an 5, Thouin annonça aux «Professeurs administrateurs du Muséum national d'Histoire naturelle à Paris» qu'il procédait à l'expédition d'environ 500 pièces " (en réalité près de 600 !). Le 14 ventôse an 6 (4 mars 1798) on lit "une lettre du Cit ${ }^{\text {en }}$ Thouin au sujet de l'envoi des poissons pétrifiés du Mont bolca du Véronois et des animaux affricains $[$ sic] arrivés à Toulon ". Cinq mois plus tard, le 14 thermidor an 6 (1 er $\left.^{\text {aout }} 1798\right)$, est mentionnée « une lettre du Ministre de l'Intérieur qui écrit que le Cen Thouin, l'un des commissaires envoyés par le gouvernement en Italie, a du [sic] faire parvenir au Muséum les objets d'histoire naturelle recueillis dans ce pays ". Enfin, le $4^{\mathrm{e}}$ jour complémentaire de l'an 6 (20 septembre 1798), il est question d' "une lettre du Ministre de l'Intérieur qui nomme le Citen Thouin commissaire pour assister, au nom du gouvernement, à l'ouverture des caisses arrivées d'Italie et renfermant des objets d'histoire naturelle destinés pour le Muséum : les Citoyens Faujas et Lacépède sont choisis par l'assemblée pour assister au nom de l'administration à cette même ouverture qui est fixée au Deux vendémiaire an sept à neuf heures du matin» (soit le 23 septembre 1798).

Une version édulcorée des évènements évoqués ci-dessus se trouve dans le tome premier de l'ouvrage que publia Faujas de Saint-Fond, titulaire de la chaire de Géologie, en 1803, sous le titre d'Essai de Géologie ou Mémoires pour servir à l'histoire naturelle du Globe. On y lit en effet (Faujas Saint-Fond 1803: 28, note 1) que "Bonaparte fit l'acquisition de la collection de Gazzola, et l'envoya au Muséum national d'histoire naturelle de Paris; elle en fait un des principaux ornements; en même temps que par le nombre, la grandeur, la variété et la belle conservation des poissons presque tous exotiques dont elle est composée, elle forme une des bases importantes de la Géologie. Gazzola, depuis cette époque, s'est procuré de nouveaux objets en ce genre, dus à son zèle et à ses infatigables recherches». Une version semblable se retrouve sous la plume de Deleuze qui dans son Histoire et Description du Muséum royal d'Histoire naturelle (1823: 345) mentionne dans sa description du laboratoire de Géologie «la suite magnifique des poissons fossiles de Monte-Bolca, rassemblée par les soins de M. le comte Gazola, de qui le gouvernement l'acquit pour le Muséum en 1798 ". La notion d' "acquisition" ne manquera évidemment pas de surprendre lorsqu'on sait qu'elle est le fruit d'une confiscation. Mais l'affaire ne s'arrête pas là, car un nouvel épisode imprévu se produisit cinq ans plus tard.

On notera ici qu'une partie des pièces reçues par le Muséum national d'Histoire naturelle ne s'y trouve plus. C'est ainsi que l'un des cinq membres du Directoire, Louis-Marie de la Révellière-Lépeaux (1753-1824), botaniste de formation, facilita l'envoi au cabinet d'Histoire naturelle d'Angers, à la demande de son fondateur, Joseph-Étienne Renou (1740-1809), de douze poissons fossiles de la collection Gazola. Il faut toutefois reconnaître que ces poissons, "dits de Bonaparte" sont de qualité médiocre. De même, il nous a été donné d'observer, dans les collections de l'université Claude Bernard de Lyon, deux poissons fossiles du Monte Bolca dont l'étiquette indique "Vestena nova dans le Veronais, du Cabinet de Gazola...». 


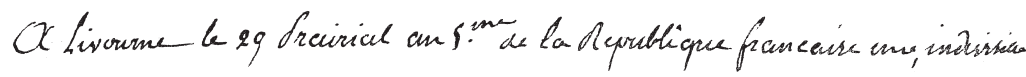

Citoyers Chers Collegues

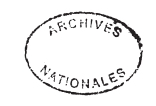

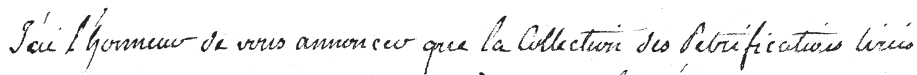
de la montagne de Verome est aequise prow la déprobligue et quéde

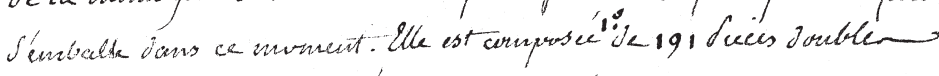
prescutant lis deno mities de chaque proison, parcorssequent 282 , Leyantitions. 20. ck 138 Suces Simples qui n offent himprisite que

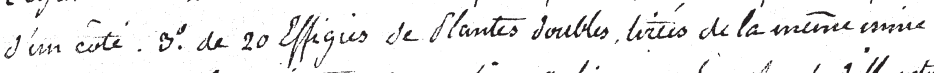

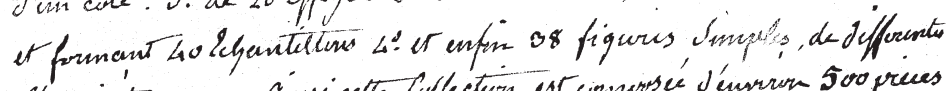

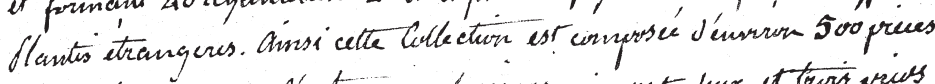

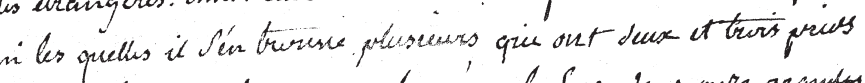
quarrés de Surface ic tort est reveforme' on le Sore dans onse grandes

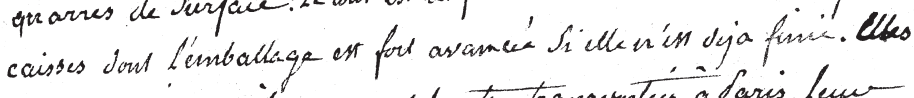
Sunot enrryeis a milan prow cela etre transpontecs a Saris. Leun-

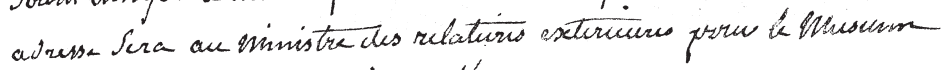
n! J'histore naturele Sieisont hisages.

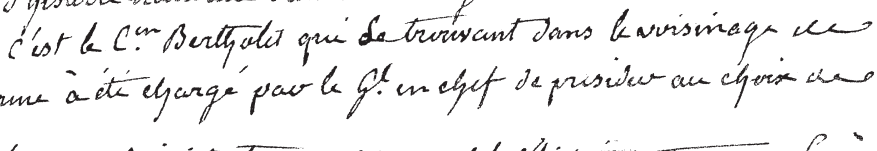

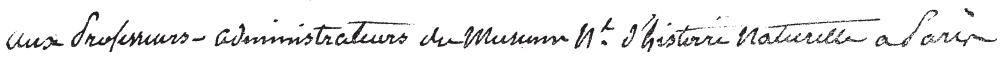

Ceste collection, à Son emballage et à Son expeedition. Il me marque.

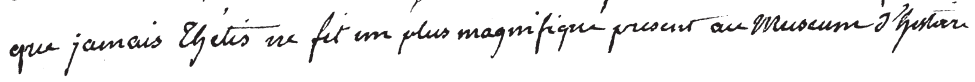
naturath.

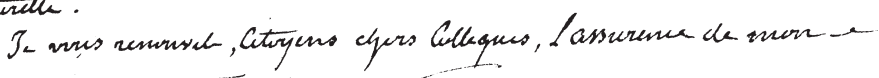
Sincere ot nespertucuor attacyenunt

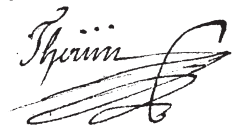

FIG. 3. - Lettre adressée de Livourne le 29 Prairial an 5 (17 juin 1797) par André Thouin aux professeurs administrateurs du Muséum national d'Histoire naturelle pour leur annoncer que «la collection de pétrifications tirées de la montagne de Véronne [sic]» est actuellement en cours d'emballage en vue de son expédition vers la France (Archives nationales; AJ 15/836). 

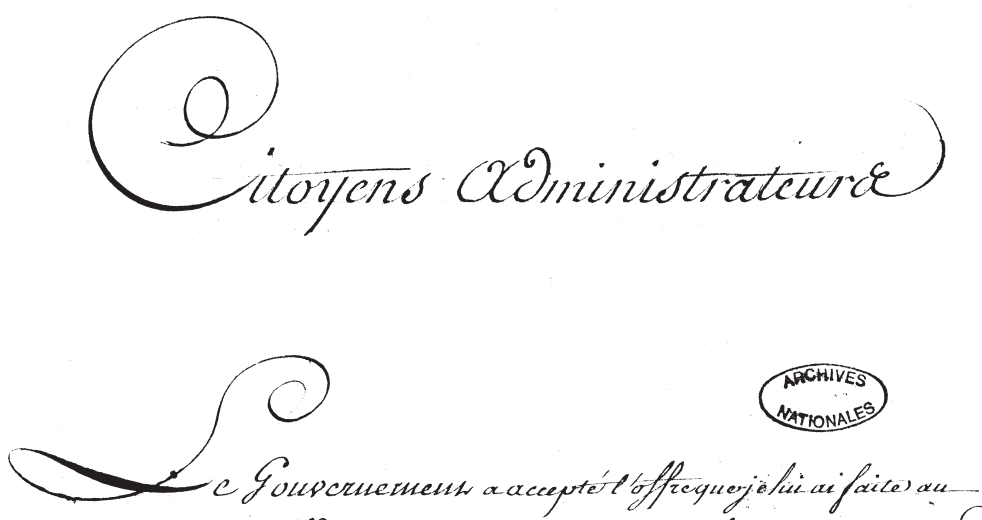

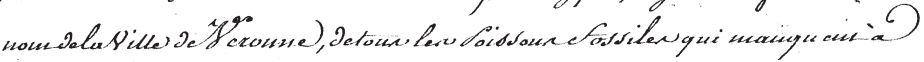
vater cullectios,

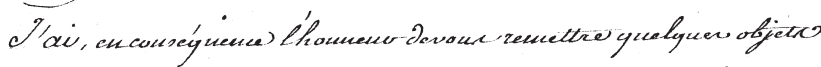

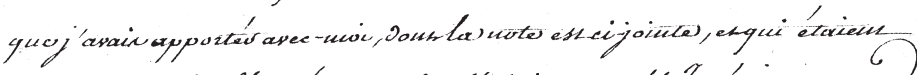

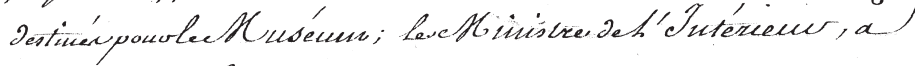

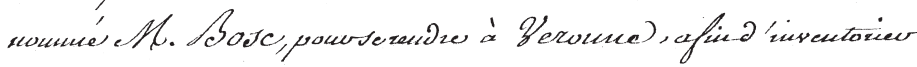
erfairer arreser cettes Collection à Sarin.

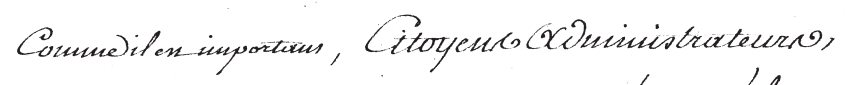

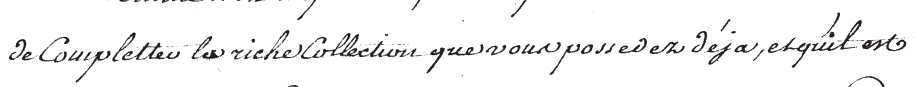

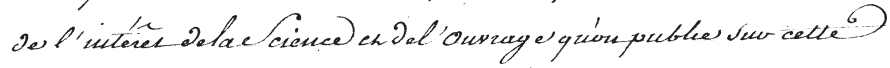

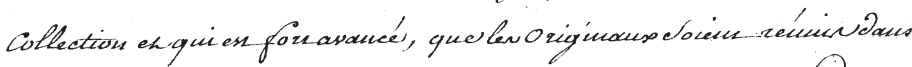

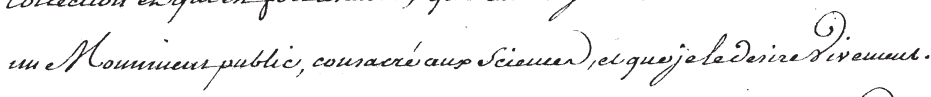

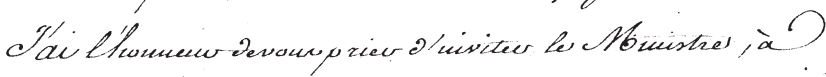

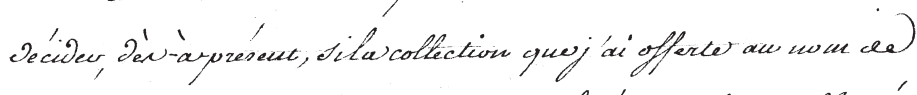

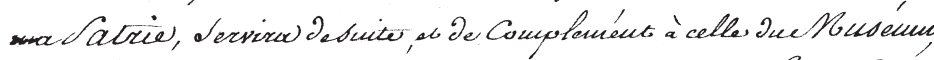

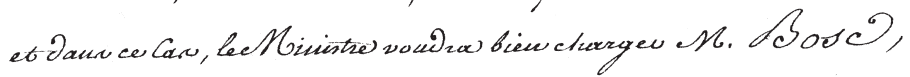

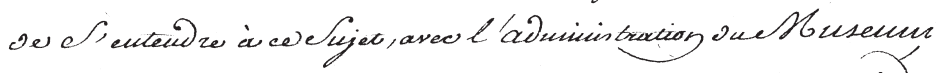

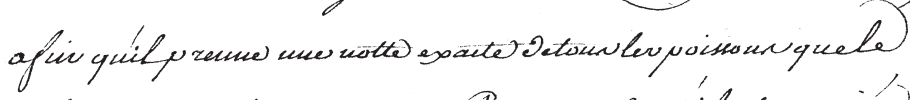

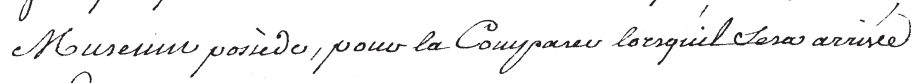

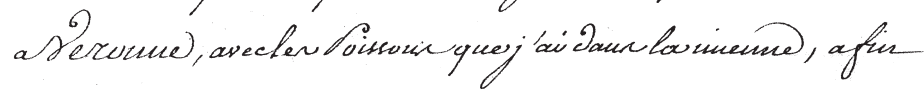

FIG. 4. - Lettre de Giovanni Battista (Jean-Baptiste) Gazola, datée du 20 Floréal an 11 (10 mai 1803) aux Citoyens administrateurs du Muséum national d'Histoire naturelle, dans laquelle il offre à cette institution de nouveaux poissons fossiles du Monte Bolca. (Archives nationales AJ 15/590). 


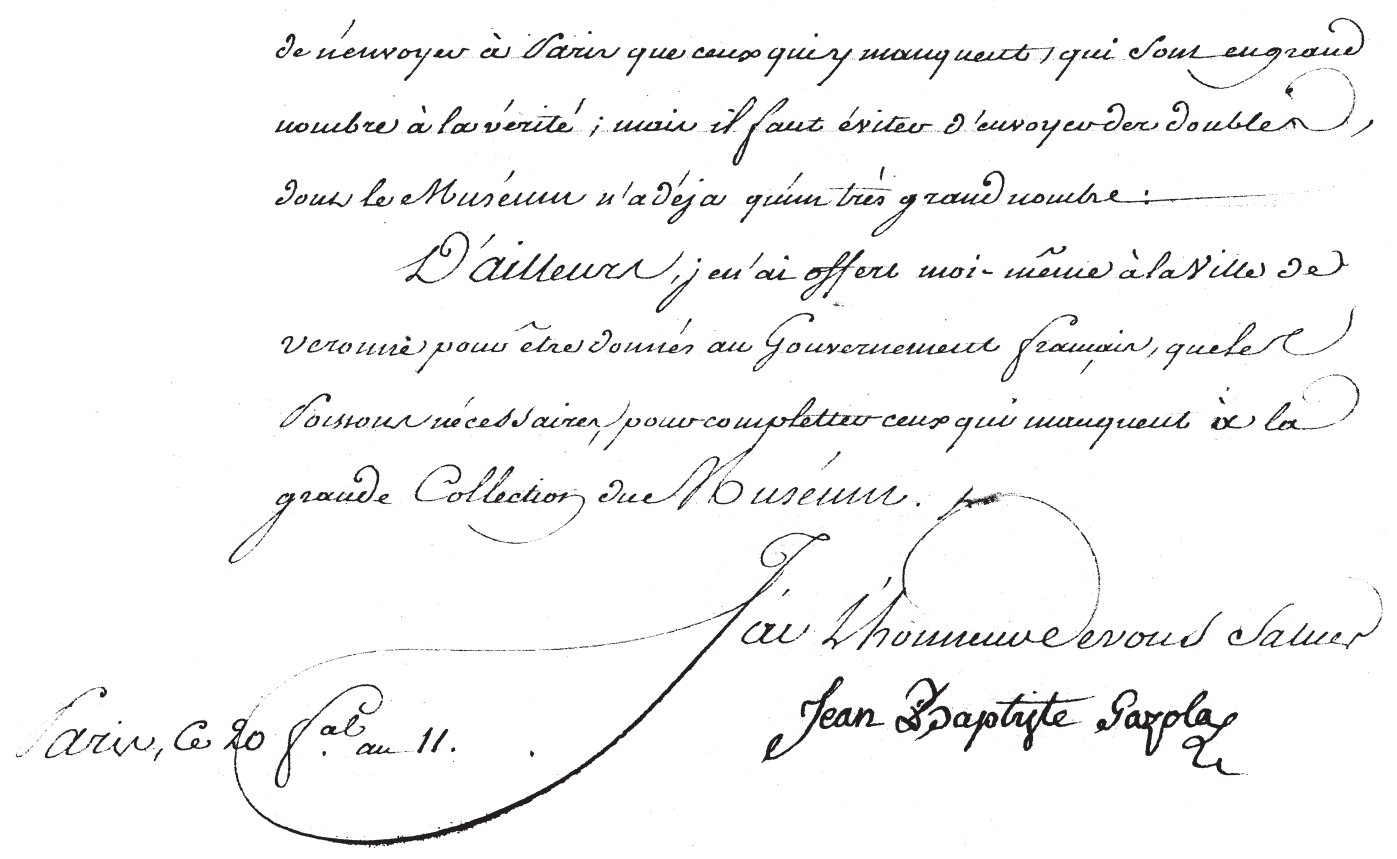

FIG. 4. - Suite.

\section{LA MAGNANIMITÉ DE GIOVANNI BATTISTA GAZOLA}

Propriétaire d'une partie du gisement de Bolca, Giovanni Battista Gazola ne se laissa pas décourager par la confiscation de la moitié de sa collection de poissons fossiles car, outre l'acquisition de la collection d'Ignazio Ronconi, il décida d'organiser de nouvelles fouilles de la Pesciara en collaboration avec le jeune Tommaso Antonio Catullo (1782-1869). D'autre part, dès le 28 octobre 1797, l'imprimeur Bartolomeo Giuliari reprit personnellement en main le projet de publication de l'Ittiolitologia Veronese. Cette poursuite des travaux d'impression fut rendue possible par le fait que les fossiles confisqués, susceptibles d'être figurés dans ce volume, avaient déjà été dessinés avant l'arrivée à Vérone des troupes françaises. Il fut donc décidé de poursuivre la préparation de l'œuvre en dépit de la confiscation des pièces de référence. En raison de nombreuses vicissitudes, l'achèvement de cette œuvre traîna en longueur, si bien que l'impression ne fut achevée qu’en décembre 1809 (Gaudant 2011).
Pour sa part, l'opportunisme de Giovanni Battista Gazola - qui avait précédemment hébergé le futur roi Louis XVIII dans sa villa de la Via del Fantefit qu'il ne tarda pas à juger favorablement certains effets de la présence française qui se traduisit par la proclamation, dès le 9 juillet 1797 d'une République cisalpine qui se transforma en 1802 en République italienne, mettant ainsi fin au régime féodal de la République de Venise. Il accepta même de présider l'administration municipale de Vérone.

Naquit alors dans son esprit l'idée d'un voyage à Paris pour offrir au Premier Consul, de la part de la commune de Vérone, de nouvelles pétrifications. En effet, il n'avait pas manqué de reconnaître, au sein du matériel nouvellement recueilli au Monte Bolca, la présence d'un certain nombre de pièces nouvelles susceptibles de compléter la partie de sa collection désormais conservée à Paris. C'est ainsi qu'il quitta Vérone à la fin de 1802 . Le procèsverbal manuscrit de l'assemblée des professeurs du Muséum national d'Histoire naturelle qui s'est tenue le 13 nivôse an 11 (3 janvier 1803) est ainsi libellé: «Notre collègue Faujas présente 
à l'assemblée Monsieur le Comte de Gazola. Le Directeur s'est empressé au nom de l'assemblée, d'accueillir ce savant illustre, et l'a invité à prendre place et à assister à la séance des Professeurs. Il nous a fait voir une superbe collection de Poissons pétrifiés disséminés dans une masse de terre argilo-calcaire venant du mont-Bolca. Ce morceau très remarquable est divisé en plusieurs feuillets réunis dans une bouëte s'ouvrant en trois parties». Cinq mois plus tard, Giovanni Battista Gazola fit remettre aux administrateurs du Muséum national d'Histoire naturelle une lettre datée du 20 Floréal an 11 (10 mai 1803) dans laquelle il dévoile toute sa grandeur d'âme car il y souligne qu' «il est important [..] de completter $[$ sic] la riche collection que vous possédez déjà, et qu'il est de l'intérêt de la science et de l'ouvrage qu'on publie sur cette collection et qui est fort avancé, que les originaux soient réunis dans un monument public, consacré aux sciences, et que je le désire vivement". Il propose donc, "au nom de [sa] Patrie» (c'est-à-dire au nom de la ville de Vérone) d'offrir une collection qui "servira de suite, et de complément à celle du Muséum" mais «de n'envoyer à Paris que ceux qui y manquent, qui sont en grand nombre à la vérité» (Figure 4).

Cette offre fut officiellement acceptée un mois plus tard et l'assemblée des professeurs prit connaissance le 26 Prairial an 11 (15 juin 1803) d'une "lettre du Ministre de l'Intérieur qui a arrêté que la collection de Poissons pétrifiés offerte au Premier Consul par la Ville de Vérone, seroit transportée à Paris, dans sa totalité ». Un autre mois s'écoula avant que le procès-verbal de la séance du 24 Messidor an 11 (13 juillet 1803) rende compte d' "une lettre de M. Gazola, qui a bien voulu se charger de l'arrangement des poissons fossiles qui jadis faisoient partie de son Cabinet à Véronne $[s i c] »$. On ne sait cependant pas à quelle date ces fossiles arrivèrent au Muséum.

Toujours est-il qu'après son retour à Vérone, Giovanni Battista Gazola rendit compte de sa mission devant le Conseil communal dans sa réunion du 29 octobre 1803, comme en atteste le procès-verbal. Et le 7 novembre 1803, le citoyen Dariff, membre du Conseil communal, crut utile de prononcer un chaleureux éloge de celui qui en était le président: "À la lecture du procès-verbal de la dernière séance, vous avez entendu, citoyens collègues, avec quelle grandeur d'âme, avec quel désintéressement, le citoyen Gio Batta Gazola, envoyé à Paris pour le bien de cette Commune, s'est efforcé de cultiver l'âme du Premier Consul et Président, en faveur de celle-ci. Il aurait pu s'arroger un mérite personnel en offrant au Héros en son nom propre ces pièces rares qui lui appartenaient; mais non : préférant généreusement à sa gloire propre celle de Vérone, il a voulu au nom de celle-ci faire un agréable présent à celui dans les mains de qui repose le bonheur de celle-ci. La Patrie laissera-t-elle sans reconnaissance une action si sublime? Quel Citoyen fera encore un sacrifice à son profit si elle néglige de récompenser au moins par une marque de gratitude et de remerciement les âmes zélées et bénévoles?».

Devenue propriété du Muséum national d'Histoire naturelle, la collection Gazola fut examinée par Henry Ducrotay de Blainville qui avait été chargé d'écrire l'article "Poissons fossiles" du Nouveau dictionnaire d'histoire naturelle (1818). C'est toutefois Louis Agassiz qui en réalisa l'étude détaillée (Agassiz 1833-1844), ce qui le conduisit à publier séparément une sévère Revue critique des Poissons fossiles figurés dans l'Ittiolitologia Veronese (Agassiz 1835) qui demeura pratiquement confidentielle alors que sa traduction allemande intitulée Kritische Revision der in der Ittiolitologia Veronese abgebildeten fossilen Fische fut publiée la même année dans le Neues Jahrbuch für Mineralogie, Geognosie, Geologie und Petrefaktenkunde.

Le catalogue manuscrit d'Anatomie comparée du Muséum national d'Histoire naturelle dressé en 1861 énumère (p. 1523-1583) sous les numéros 10693 à 11024 les «Poissons fossiles du Calcaire pisolitique de Monte Bolca (Vestena nova) dans le Veronais - Collection Gazola, acquise en 1797 par le Général Bonaparte et donnée par lui au Muséum ». Leur nombre s'élevait alors à 332. Ces fossiles étaient conservés dans les armoires $n^{\circ}$ LIX à LXIV.

Ultérieurement, Charles R. Eastman en révisa les types dans un article sobrement intitulé Descriptions of Bolca Fishes (Eastman 1904). 


\section{DESTINÉE DE LA NOUVELLE COLLECTION GAZOLA}

À la mort de Giovanni Battista Gazola, survenue en 1834, sa seconde collection passa entre les mains de son neveu Giovanni, lequel chargea en 1843 Giuseppe Cerato de poursuivre l'exploitation du gisement de Bolca.

Nicolis (1893): d'après le catalogue manuscrit préparé par le professeur Antonio Manganotti, «la collection comporte environ 1200 exemplaires pratiquement tous en excellent état de conservation beaucoup d'entre eux comportant l'empreinte et la contre-empreinte - artistiquement disposés dans d'élégants rayonnages et cadres».

\section{Remerciements}

L'auteur remercie vivement Madame Bruna Burato (Vérone), qui lui a aimablement communiqué la photographie du portrait de Giovanni Battista Gazola conservé au Museo civico di Storia naturale de Vérone, ainsi que P. Taquet (Muséum national d'Histoire naturelle, Paris) et G. Carnevale (Université de Pise, Italie), pour leurs remarques constructives.

\section{RÉFÉRENCES}

\section{SOURCES MANUSCRITES}

\section{Archives nationales}

AJ 15: Muséum national d'Histoire naturelle - Assemblées des professeurs, minutes des procèsverbaux:

dossiers AJ 15/98 (Vendémiaire an V-Prairial an VI);

AJ 15/589 (Vendémiaire an XI- Ventose An XI); AJ 15/590 (Germinal an XI- Fructidor an XI); - Correspondance (arrivée et départ) : dossiers AJ 15/742 et AJ 15/743;

- Collections diverses: collections d'objets recueillis en Italie (an V-an VI) : dossier AJ 15/836;

- Anatomie Comparée: catalogue des ossements fossiles de Vertébrés placés dans les galeries de Géologie et Minéralogie. Volume deuxième (ce catalogue manuscrit est conservé dans l'Unité de gestion des collections de Paléontologie du Muséum national d'Histoire naturelle).

\section{F 17: Procès-verbaux de la Commission tempo-} raire des Arts :

Dossier F 17/1275 A (Commission pour la recherche des objets de sciences et arts en Italie) : sousdossier $n^{\circ} 2$.

\section{SOURCES IMPRIMÉES}

Agassiz L. 1833-1844. - Recherches sur les Poissons fossiles. Petitpierre, Neuchâtel, 5 vol., Atlas.

Agassiz L. 1835. - Revue critique des Poissons fossiles figurés dans l'tttiolitologia Veronese. Petitpierre et Prince, Neuchâtel, 44 p.

BlainVILle H. DuCROTAY DE 1818. — Poissons fossiles. In Nouveau Dictionnaire d'Histoire naturelle appliquée aux arts... Deterville, Paris, XXVII: 310-395.

Cipolla C. 1954. - La storia politica di Verona. Edizioni Valdonega, Verona, $299 \mathrm{p}$.

DARU P.-B. 1821. - Histoire de la République de Venise. T. VIII. Firmin-Didot, Paris, 643 p.

Deleuze M. 1823. - Histoire et description du Muséum national d'Histoire naturelle. M. A. Royer, Paris, 2 vol., $\mathrm{VI}+720 \mathrm{p}$.

EASTMAN C.R. 1904. - Descriptions of Bolca Fishes. Bulletin of the Museum of Comparative Zoology at Harvard College, XLVI, 1: 1-36.

Faujas ST-Fond B. 1803. - An XI. Essai de Géologie ou Mémoires pour servir à l'histoire naturelle du Globe. T. 1. C. F. Patris, Paris, 493 p.

Frigo M. \& Sorbini L. 1997. - 600 fossili per $\mathrm{Na}$ poleone. Museo Civico di Storia naturale di Verona, Verona, $31 \mathrm{p}$.

GAUDANT J. 2011. - La publication de l'Ittiolitologia Veronese (1796-1809): le triomphe de l'obstination au service d'une entreprise scientifique hors norme. Studi e Ricerche sui giacimenti terziari di Bolca 13: 67-133.

Nicolis E. 1893. - Del Museo Gazoliano - Relazione allillustrissimo Avv. Comm. Augusto Caperle Sindaco di Verona. Stabilimento tipo-litografico G. Franchini, Verona, $14 \mathrm{p}$.

VolTA G. S. 1789. - Deglimpietrimenti del Territorio veronese ed in particolare dei pesci fossili del celebre Monte Bolca per servire di continuazione all'argomento delle rivoluzione terracquee. s.n.: 1-24.

VOLTA G. S. 1796-1809. — Ittiolitologia Veronese del Museo Bozziano ora anesso a quello del Conte Giovambattista Gazola e di altri gabinetti di fossili veronesi. Dalla Stamperia Giuliari, Verona, p. I-LII + I-CCCXXIII, pls I-LXXVI.

Soumis le 11 mai 2010; accepté le 3 janvier 2011. 